\title{
Modeling and forecasting call center arrivals: a literature survey and a case study
}

\author{
Rouba Ibrahim \\ School of Management, University College London \\ rouba.ibrahim@ucl.ac.uk \\ Han Ye \\ Business Administration, University of Illinois at Urbana Champaign \\ hanye@illinois.edu \\ Pierre L'Ecuyer \\ Computer Science and Operations Research, University of Montreal \\ lecuyer@iro.umontreal.ca \\ Haipeng Shen \\ Statistics and Operations Research, University of North Carolina at Chapel Hill \\ haipeng@email.unc.edu
}

\begin{abstract}
The effective management of call centers is a challenging task mainly because managers are consistently facing considerable uncertainty. Among important sources of uncertainty are call arrival rates which are typically time-varying, stochastic, dependent across time periods and across call types, and often affected by external events. Accurately modeling and forecasting future call arrival volumes is a complicated issue which is critical for making important operational decisions, such as staffing and scheduling, in the call center. In this paper, we review the existing literature on modeling and forecasting call arrivals. We also discuss the key issues in building good statistical arrival models. Additionally, we evaluate the forecasting accuracy of selected models in an empirical study with real-life call center data. We conclude by summarizing future research directions in this important field.
\end{abstract}

Keywords: Call center arrivals, Forecasting, Time series, Doubly stochastic Poisson, Fixed-effects, Mixed-effects, ARIMA, Exponential smoothing, Bayesian, Dimension reduction, Dependence, Seasonality, Marketing events

\section{Introduction}

The call center services industry is large and important with more than 2.7 million agents working in the United States and 2.1 million agents working at Europe, the Middle East, and Africa (Akşin et al., 2007). Efficiently managing a call center is a challenging task because managers 
have to make staffing and scheduling decisions to balance between staffing cost and service quality, which always contradict, in the presence of uncertain arriving demand. Most staffing or scheduling plans start with forecasting customer call arrivals, which are highly stochastic. Accurately forecasting call arrivals is one of the keys to achieve optimal operational efficiency, since under-forecasting leads to under-staffing and then long customer waiting, while over-forecasting results in a waste of money on over-staffing.

The process of customer arrivals is nontrivial. This process can be modeled as a Poisson arrival process and has been shown to possess several features (Akşin et al., 2007; Cez̧ik and L'Ecuyer, 2008; Gans et al., 2003; Garnett et al., 2002; Wallace and Whitt, 2005). One of the most important features is that the arrival rate is time varying, which adds complexity to the forecasting process. Call arrival rates may exhibit intraday, weekly, monthly, and yearly seasonalities. While a time-inhomogeneous Poisson arrival process can easily capture time dependence in call arrival data, it often fails to capture other characteristics. For one, call center arrivals typically exhibit significant dispersion relative to the Poisson distribution. Thus, a doubly stochastic Poisson arrival process may be more appropriate, e.g., see Aldor-Noiman et al. (2009); Avramidis et al. (2004); Ding and Koole (2015); Ibrahim and L'Ecuyer (2013). For two, call center arrivals also exhibit different types of dependencies including intraday (withinday), interday, and inter-type dependence, e.g., see Aldor-Noiman et al. (2009); Avramidis et al. (2004); Channouf and L'Ecuyer (2012); Shen and Huang (2008b); Tanir and Booth (1999); Whitt (1999b). A reasonable forecasting model needs to appropriately account for some or all types of dependencies that exist in real data.

In the presence of intraday and interday dependence of call arrival rates, standard time series models may be applied to forecast call arrivals, for example autoregressive integrated moving average (ARIMA) models and exponential smoothing (Hyndman et al., 2008). In addition, some recent papers have proposed fixed-effects models (Ibrahim and L'Ecuyer, 2013; Shen and Huang, 2008b; Taylor, 2008; Weinberg et al., 2007) and mixed-effects models (Aldor-Noiman et al., 2009; Ibrahim and L'Ecuyer, 2013) to account for within-day dependence, interday dependence, and inter-type dependence of call arrivals. Dimension reduction (Shen and Huang, 2005, 2008b,a) or Bayesian techniques (Aktekin and Soyer, 2011; Soyer and Tarimcilar, 2008; Weinberg et al., 2007) are also adopted in the existing literature.

The remainder of the paper is organized as follows. Key features of call center arrival processes are discussed in Section 2. Forecasting methods which are proposed in the existing literature are examined in Section 3. A case study in which several methods from the recent literature are compared is reported in Section 4, using a Canadian call center data set, which reveals the practical features of those methods. Discussions of future research directions are offered in Section 5 to conclude the paper. The conference paper Ibrahim et al. (2012) served as a starting point for this survey.

\section{Key Properties of Call Center Arrival Processes}

A natural model for call arrivals is the Poisson arrival process (Akşin et al., 2007; Cez̧ik and L'Ecuyer, 2008; Gans et al., 2003; Garnett et al., 2002; Wallace and Whitt, 2005). This model is theoretically justified by assuming a large population of potential customers where each customer independently makes a call with a very small probability; the total number of calls made in a given time interval is then approximately Poisson. As mentioned in Kim and Whitt (2014a), the so-called Poisson superposition theorem is a supporting limit theorem, e.g., see Barbour et al. (1992). 


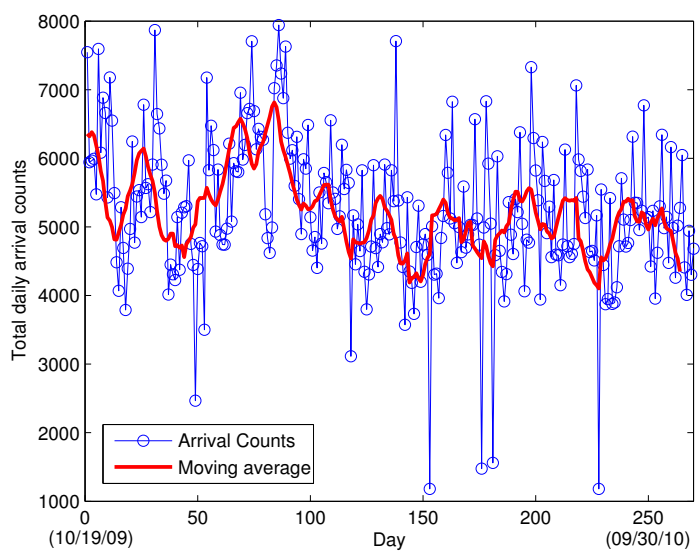

Figure 1: Daily call arrival counts over successive months in a Canadian call center.

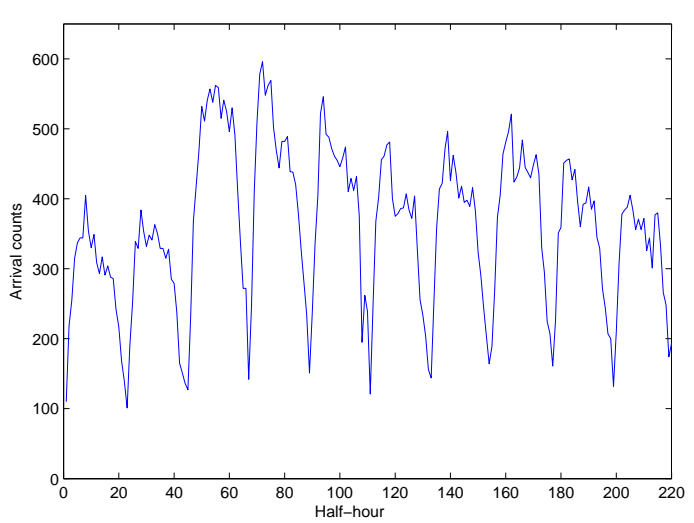

Figure 2: Half-hourly call arrival counts over two consecutive weeks.

Recent empirical studies have shown multiple important properties of the call arrival process, many of which are not consistent with the Poisson modeling assumption. In this section, we describe those properties in detail; for a more abridged description, see $\S 2$ in Ibrahim et al. (2012).

Time dependence of call arrival rates.. One of the most important properties of call arrival rates is that they vary with time. In particular, call arrival rates typically exhibit intraday (withinday), daily, weekly, monthly, and yearly seasonalities. We illustrate this time-dependence property in Figures 1, 2, and 3 (taken from Ibrahim and L'Ecuyer (2013)), which show arrival patterns that are commonly observed in call centers.

In Figure 1, we plot the number of calls per day arriving to the call center of a Canadian company between October 19, 2009 and September 30, 2010. Figure 1 shows that there exist monthly fluctuations in the data. For example, the moving average line in the plot, which is computed for each day as the average of the past 10 days, suggests that there is an increase in call volume during the months of January and February, i.e., days 54 to 93 in the plot.

In Figure 2, we illustrate weekly seasonality by plotting daily arrival counts, of the same call type as in Figure 1, over two consecutive weeks in the call center. The call center is closed on weekends, so we have a total of 10 workdays in the plot. Figure 2 clearly shows that there is a strong weekly seasonality in the data. Such weekly patterns are very commonly observed in practice, e.g., see Figure 1 in Taylor (2008) and Figure 2 in Taylor (2012).

For a more microscopic view of arrivals, we plot half-hourly average arrival counts, per weekday, in Figure 3. These intraday averages constitute the daily profile of call arrivals. Figure 3 shows that call volumes are higher, on average, on Mondays than on the remaining weekdays. Figure 3 also shows that all weekdays have a similar daily profile: there are two major daily peaks for call arrivals. The first peak occurs in the morning, shortly before 11:00 AM, and the second peak occurs in the early afternoon, around 1:30 PM. (There is also a third "peak", smaller in magnitude, which occurs shortly before 4:00 PM on Mondays, Tuesdays, and Wednesdays.) Such intraday arrival patterns are also characteristic of call center arrivals; e.g., see AldorNoiman et al. (2009); Avramidis et al. (2004); Channouf et al. (2007); Gans et al. (2003); Tanir and Booth (1999).

Given that arrival rates are time-varying, which is not accounted for in a Poisson arrival process, 


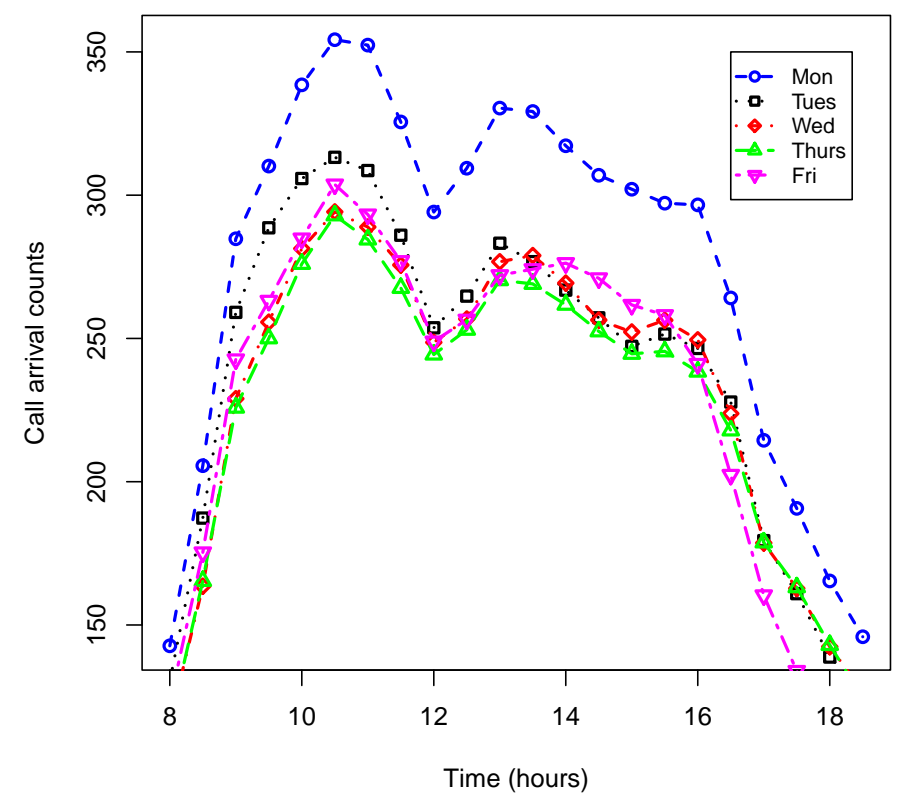

Figure 3: Intraday profile of call arrival counts per weekday in a Canadian call center.

a natural extension is to consider a nonhomogeneous Poisson process with a deterministic and time-varying arrival-rate function. For simplicity, it is commonly assumed that call arrival rates are constant in consecutive 15 or 30 minute intervals during a given day; e.g., see Brown et al. (2005); Green et al. (2007); Liao et al. (2012).

Nevertheless, it is important to perform statistical tests to confirm that it is appropriate to model call center data as a nonhomogeneous Poisson process. Brown et al. (2005) proposed a specific test procedure based on a Kolmogorov-Smirnov test and did not reject the null hypothesis that arrivals of calls are from a nonhomogeneous Poisson process with piecewise constant rates. Kim and Whitt (2014b) examined several alternative test procedures which have greater power compared to the one suggested in Brown et al. (2005). Kim and Whitt (2014a) applied Kolmogorov-Smirnov tests to banking call center and hospital emergency department arrival data and showed that they are consistent with the nonhomogeneous Poisson property, but only if certain common features of the data have been accounted for, including: data rounding, interval partition, and overdispersion caused by combining data.

Overdispersion of arrival counts.. A consequence of the Poisson modeling assumption is that the variance of the arrival count in each time period is equal to its expectation during that period. However, there is empirical evidence which invalidates this assumption. Indeed, it has been observed that the variance of an arrival count per time period is usually much larger than its expected value; see Aldor-Noiman et al. (2009); Avramidis et al. (2004); Jongbloed and Koole (2001); Steckley et al. (2005). One way of dealing with this overdispersion of count data is to assume that the Poisson arrival process is doubly stochastic, i.e., that the arrival rate itself is a stochastic process; e.g., see Aldor-Noiman et al. (2009); Avramidis et al. (2004); Ibrahim and L'Ecuyer (2013); Jongbloed and Koole (2001); Shen (2010b); Soyer and Tarimcilar (2008); Steckley et al. (2004); Weinberg et al. (2007). 
A doubly stochastic Poisson process can be viewed as a two-step randomization: A stochastic process (for the arrival rate) is used to generate another stochastic process (for the call arrival process) by acting as its intensity. We now illustrate why a doubly stochastic Poisson process is a way to deal with a higher variance in the arrival count data. Denote by $X_{j}$ the number of arrivals in a given period $j$, and let $\Lambda_{j}$ denote the cumulative arrival rate (its integral) over period $j$. Then, assume that conditional on $\Lambda_{j}, X_{j}$ has a Poisson distribution with mean $\Lambda_{j}$. To simplify notation, we assume in this paper that all periods have the same length and also that the time unit is equal to one period. Then, when the arrival rate is constant over each period, this rate is the same as the cumulative rate $\Lambda_{j}$, and we denote both by $\Lambda_{j}$. By conditioning on $\Lambda_{j}$, the variance of $X_{j}$ is given by:

$$
\operatorname{Var}\left[X_{j}\right]=\mathbb{E}\left[\operatorname{Var}\left[X_{j} \mid \Lambda_{j}\right]\right]+\operatorname{Var}\left[\mathbb{E}\left[X_{j} \mid \Lambda_{j}\right]\right]=\mathbb{E}\left[\Lambda_{j}\right]+\operatorname{Var}\left[\Lambda_{j}\right]
$$

With a random arrival rate function, we have that $\operatorname{Var}\left[\Lambda_{j}\right]>0$ on the right-hand side of (1), which accounts for the additional variance in $\operatorname{Var}\left[X_{j}\right]$.

Maman et al. (2015) study the implications of assuming a doubly stochastic Poisson process on operational decision making in the system. In particular, to model the doubly stochastic Poisson process, Maman et al. (2015) proposed a Poisson mixture model with a parametric form of the random Poisson arrival rate. They then incorporated the Poisson mixture model into a queuing model and derived asymptotic optimal staffing levels.

Zhang et al. (2014) show through statistical analysis that the level of stochastic variation of the arrival process is neither as low as in a standard Poisson process, nor as high as in a doubly stochastic process. They propose a model to control for this level of overdispersion. Glynn et al. (2014) show that timescale is the key that underlies the different conclusions in the literature regarding the Poissonness of the arrivals. In particular, they show that the arrival process is Poisson-like on a short timescale (minutes), but not so on longer timescales (hours, days, etc.). The effect of time scale is also extensively examined in Oreshkin et al. (2014).

Interday and Intraday dependencies.. In real-life call centers, there is typically evidence for dependencies between the arrival counts, or arrival rates, in different time periods within a single day, or across several days; e.g., see Aldor-Noiman et al. (2009); Avramidis et al. (2004); Channouf and L'Ecuyer (2012); Shen and Huang (2008b); Tanir and Booth (1999); Whitt (1999b). Those interday (day-to-day) and intraday dependencies typically remain strong even after correcting for detectable seasonalities. Indeed, it is important to do such a correction to avoid erroneously overestimating dependencies in the data.

In Tables 1 and 2 we illustrate interday and intraday correlations in the same call center as in Figures 1-3. Tables 1 and 2 illustrate several properties which are very commonly observed in practice: (i) correlations are strong and positive between successive weekdays; (ii) interday correlations are slightly smaller with longer lags; (iii) Mondays are less correlated with the remaining weekdays; (iv) correlations are strong and positive between successive half-hourly periods within a day; and (v) intraday correlations are slightly smaller with longer lags.

There are different measures that could be used to capture interday and intraday dependencies in call arrival data. The most commonly used measure is Pearson's correlation coefficient which captures linear dependence in the data; e.g., see Aldor-Noiman et al. (2009); Avramidis et al. (2004); Ibrahim and L'Ecuyer (2013); Shen and Huang (2008b). However, since dependencies may not be linear, it is also useful to consider alternative measures such as rank correlation coefficients; see Channouf and L'Ecuyer (2012) and references therein. For example, Spearman's 


\begin{tabular}{c|ccccc}
\hline Weekday & Mon & Tues. & Wed. & Thurs. & Fri. \\
\hline Mon. & 1.0 & 0.48 & 0.35 & 0.35 & 0.34 \\
Tues. & & 1.0 & 0.68 & 0.62 & 0.62 \\
Wed. & & & 1.0 & 0.72 & 0.67 \\
Thurs. & & & & 1.0 & 0.80 \\
Fri. & & & & & 1.0 \\
\hline
\end{tabular}

Table 1: Correlations between arrival counts on successive weekdays in a Canadian call center.

\begin{tabular}{c|ccccc}
\hline Half-hour periods & $(10,10: 30)$ & $(10: 30,11)$ & $(11,11: 30)$ & $(11: 30,12)$ & $(12,12: 30)$ \\
\hline$(10,10: 30)$ & 1.0 & 0.87 & 0.80 & 0.73 & 0.66 \\
$(10: 30,11)$ & & 1.0 & 0.82 & 0.74 & 0.71 \\
$(11,11: 30)$ & & & 1.0 & 0.83 & 0.80 \\
$(11: 30,12)$ & & & & 1.0 & 0.81 \\
$(12,12: 30)$ & & & & & 1.0 \\
\hline
\end{tabular}

Table 2: Correlations between arrivals in consecutive half-hour periods on Wednesday morning in a Canadian call center.

rank correlation coefficient measures how well the relationship between two variables can be described using a monotonic, but not necessarily linear, function.

Mixed-effects models (Aldor-Noiman et al., 2009; Ibrahim and L'Ecuyer, 2013) and, more generally, copulas (Channouf and L'Ecuyer, 2012; Jaoua et al., 2013) are ideally suited to easily capture interday and intraday dependencies in call center arrival data. Models that fail to account for positive interday and intraday dependence in call arrivals may give an overoptimistic view of call center performance measures, and the resulting errors can be very significant; see Avramidis et al. (2004); Avramidis and L'Ecuyer (2005); Steckley et al. (2005, 2009).

Inter-type dependencies.. In multi-skill call centers, there may be positive dependencies between the arrival counts, or arrival rates, corresponding to different call types. For one example, this could occur in multilingual call centers where the same service request is handled in two or several languages. For another example, this may be due to promotions or advertisements which affect several services offered by the same call center. Neglecting dependencies between different call types may lead to overloads, particularly when the same agent handles several correlated call types.

In Table 3 (taken from Ibrahim and L'Ecuyer (2013)), we present estimates of correlations between half-hourly arrival counts for two different call types, Type A and Type B. In Table 3, we focus on the same consecutive half-hour periods as in Table 2. Table 3 illustrates that intertype correlations can be strong and positive. Here, call arrivals to the Type A queue originate in the province of Ontario, and are mainly handled in English, whereas arrivals to the Type $\mathrm{B}$ queue originate in the province of Quebec, and are mainly handled in French. Otherwise, 


\begin{tabular}{|c|c|c|c|c|c|}
\hline $\begin{array}{ll}\text { Type B } & \text { Type A } \\
\end{array}$ & $(10,10: 30)$ & $(10: 30,11)$ & $(11,11: 30)$ & $(11: 30,12)$ & $(12,12: 30)$ \\
\hline$(10,10: 30)$ & 0.75 & 0.72 & 0.67 & 0.60 & 0.59 \\
\hline$(10: 30,11)$ & 0.76 & 0.73 & 0.72 & 0.64 & 0.62 \\
\hline$(11,11: 30)$ & 0.66 & 0.65 & 0.67 & 0.67 & 0.63 \\
\hline$(11: 30,12)$ & 0.60 & 0.56 & 0.63 & 0.63 & 0.63 \\
\hline$(12,12: 30)$ & 0.58 & 0.54 & 0.58 & 0.65 & 0.62 \\
\hline
\end{tabular}

Table 3: Correlations between Type A and Type B arrivals in consecutive half-hour periods on Wednesday in a Canadian call center.

arrivals to both queues have similar service requests. Thus, it is reasonable that there exist correlations between their respective arrival processes. There has been some recent effort to model inter-type dependencies in the data; see Ibrahim and L'Ecuyer (2013) and Jaoua et al. (2013).

Using auxiliary information.. Auxiliary information is often available in call centers to improve point or distributional forecasts considerably. For example, when a company sends notification letters to customers, or makes advertisements, this may trigger a large volume of calls; see Landon et al. (2010). Also, large sporting events or festivals can bring a significant increase of calls to emergency systems; see Channouf et al. (2007).

The past service level in the call center may also be a valuable source of information for predicting future arrivals. For example, long previous delays may lead to a high call abandonment rate, which in turn may lead to more redials in the future. Moreover, when the quality of service is poor, callers may not have their problems resolved during the first call that they make, and they may need to reconnect later. Ignoring such redials and reconnects may lead to considerably underestimating call arrival counts; see Ding et al. (2015).

Finally, in certain types of call centers, for example where people may call to report power outages or those designated to emergency services, bursts of high arrival rates over short periods of time do occur. In this context, an important accident may trigger several dozen different calls within a few minutes, all related to the same event, resulting in a much larger than expected number of calls during that time frame; e.g., see Kim et al. (2012) for the modeling of peak periods in a rural electric cooperative call center.

In recent years, there have been a few studies on forecasting call arrivals. In $\S 3$ we review the relevant literature.

\section{Call Forecasting Approaches}

In practice, arrival forecasts are needed for various purposes such as long-term capacity planning and short-term scheduling. Therefore, the specifics of the forecasting procedure need to be carefully determined, including the forecasting horizon (for time intervals, for a day, or for multiple days), and whether to combine arrivals from separate queues. Typically models that incorporate time dependencies are useful for short-term decision making since such dependencies 
tend to vanish over the longer time scale. Over a longer time horizon, simple smoothing or moving average models seem sufficient to capture the general trends in the data. Indeed, arrivals to different call types are often combined in practice when there is insufficient data for the individual call types. However, this is usually not done systematically, and is typically solely based on the experience of the call center managers.

Ideally, we want arrival models that seek to reconcile several objectives. For an arrival model to be realistic, it needs to reproduce the properties that we described in section 2. Simultaneously, for an arrival model to be practically useful, it needs to be computationally tractable. That is, it needs to rely on a relatively small number of parameters so as to avoid overfitting. Moreover, these parameters need to be easy to estimate from historical data. Finally, parameter estimates should not be hard to update (e.g., via Bayesian methods) based on newly available information, e.g., throughout the course of a day. These updated estimates would then be used to update operational decisions in the call center.

In this section, we review alternative models proposed in the literature which aim to reconcile those objectives. We first review early papers which rely mostly on standard forecasting methods (§3.1). Then, we focus on more recent models for arrivals over several days or months (§3.2). Finally, we move to models for arrivals over a single day (§3.3).

\subsection{Standard Forecasting Techniques}

The early work on forecasting call arrivals usually focused on modeling daily or even monthly total call volumes. Part of the reason for this is due to the lack of relevant data. In addition, only point forecasts of future arrival rates or counts were produced.

One of the earliest papers on forecasting call arrivals is Thompson and Tiao (1971), where the authors modeled monthly call arrivals for two different call types. Interestingly, they noted that there may be an interdependence between the arrival streams of these two call types, but they did not explore this issue further. They used seasonal autoregressive integrated moving average (ARIMA) models to forecast future call volumes, and relied solely on the past history of call arrivals in their models.

In Mabert (1985), Mabert relied on multiplicative and additive regression models, including covariates for special events and different seasonalities, to forecast daily call arrivals to an emergency call center. He also considered model adjustments which exploit previous forecasting errors to yield more accurate forecasts. He found that such models yield the most accurate forecasts, and are superior to ARIMA models.

Other early papers also relied on standard time series models. For example, Andrews and Cunningham (1995) modeled daily call arrivals to the call center of a retailer. The authors considered ARIMA models with transfer functions and incorporated covariates for advertising and special-day effects. They showed that using such information can dramatically improve the accuracy of their forecasts, and may have a significant impact on the operational decisionmaking in the call center. Similarly, Bianchi et al. (1998) used ARIMA models with intervention analysis to forecast telemarketing call arrivals. In this paper, the authors found that such models are superior to additive and multiplicative Holt-Winters exponentially weighted moving average models.

More recently, Antipov and Maede (2002) modeled the daily number of applications for loans at a financial services telephone call center. The authors also went beyond standard ARIMA models by including advertising response and special calender effects; they did so by adding 
exogenous variables in a multiplicative model. In Channouf et al. (2007), the authors developed simple additive models for the (small) number of ambulance calls during each hour, in the city of Calgary. Their models capture daily, weekly, and yearly seasonalities, selected second-order interaction effects (e.g., between the time-of-day and day-of-week), special-day effects (such as the Calgary Stampede which leads to increased call volumes), and autocorrelation of the residuals between successive hours. Their best model outperforms a doubly-seasonal ARIMA model for the residuals of a model which captures only special-day effects.

To evaluate the accuracy of forecasting models, several measures of forecasting errors have been used in the existing literature, including root mean squared error (RMSE), mean squared error (MSE), mean absolute percentage error (MAPE), weighted absolute percentage error (WAPE), etc. Which error measure is appropriate depends on the objective of call center management. For example, Ding and Koole (2015) studied a call center staffing problem where the costs are the initial staffing costs plus the intraday traffic management costs, and concluded that the optimal forecast methods should minimize the WAPE.

\subsection{Models Over Several Days}

To describe more recent arrival modelling approaches, we need some additional notation. Let $X_{i, j}$ denote the number of call arrivals during period $j, 1 \leq j \leq P$, of day $i, 1 \leq i \leq D$. The standard assumption is that call arrivals follow a Poisson process with a (potentially) random arrival rate $\Lambda_{i, j}$, which is taken to be constant over each period $j$. The cumulative arrival rate over period $j$ is also $\Lambda_{i, j}$ if a unit time period is assumed. Thus, conditional on the event $\Lambda_{i, j}=\lambda_{i, j}, X_{i, j}$ is Poisson distributed with rate $\lambda_{i, j}$.

Several papers (Aldor-Noiman et al., 2009; Brown et al., 2005; Ibrahim and L'Ecuyer, 2013; Weinberg et al., 2007) exploit the following "root-unroot" variance-stabilizing data transformation:

$$
Y_{i, j} \equiv\left(X_{i, j}+1 / 4\right)^{1 / 2} .
$$

Conditional on the event $\Lambda_{i, j}=\lambda_{i, j}$, and for large values of $\lambda_{i, j}, Y_{i, j}$ is approximately normally distributed with mean $\sqrt{\lambda_{i, j}}$ and variance $1 / 4$; see Brown et al. (2010). The unconditional distribution, with random $\Lambda_{i, j}$, is then a mixture of such normal distributions; therefore, it has a larger variance. Nevertheless, it can be assumed (as an approximation) that the squareroot transformed counts $Y_{i, j}$ are normally distributed, particularly if $\operatorname{Var}\left[\Lambda_{i, j}\right]$ is not too large. The resulting normality is very useful because it allows fitting linear Gaussian fixed-effects and mixed-effects models to the square-root transformed data.

A better alternative than modelling the arrival counts $X_{i, j}$ would be to model the rates $\Lambda_{i, j}$ directly. The reason is that it is considerably easier to simulate the system with a distributional forecast for the rates rather than one for the counts. Indeed, to simulate arrivals based on a distributional forecast for counts, one has to generate the number of arrivals in each period, and then generate the arrival times by splitting the counts uniformly and independently over the given time period. (This is consistent with the Poisson assumption.) In contrast, given a distributional forecast for the rates, one can generate the arrival times directly. Nevertheless, most arrival models in the literature are for the counts $X_{i, j}$, rather than the rates $\Lambda_{i, j}$. The reason being that, in practice, we do not observe the arrival rates themselves but only the counts which give only partial information on the rates. This makes estimating arrival rates a more complicated task.

Multiple papers, such as Ibrahim and L'Ecuyer (2013); Shen and Huang (2008b); Taylor (2008); Weinberg et al. (2007), consider a linear fixed-effects (FE) model as a benchmark for comparison. 
To illustrate, let $d_{i}$ be the day-of-week of day $i$, where $i=1,2, \ldots, D$. That is, $d_{i} \in\{1,2,3,4,5\}$ where $d_{i}=1$ denotes a Monday, $d_{i}=2$ denotes a Tuesday, ..., and $d_{i}=5$ denotes a Friday. In Ibrahim and L'Ecuyer (2013), the authors considered the following fixed-effects model for the square-root transformed arrival counts:

$$
y_{i, j}=\alpha_{d_{i}}+\beta_{j}+\theta_{d_{i}, j}+\mu_{i, j},
$$

where the coefficients $\alpha_{d_{i}}, \beta_{j}$, and $\theta_{d_{i}, j}$ are real-valued constants that need to be estimated from data, and $\mu_{i, j}$ are independent and identically distributed (i.i.d.) normal random variables with mean 0. Ibrahim and L'Ecuyer (2013) and Taylor (2008) have shown that it is difficult to beat fixed-effect models in terms of accuracy of long-term point forecasting (e.g., 2 weeks or more). Nevertheless, for short-term forecasting, one can exploit interday and intraday dependencies in the data to obtain more accurate forecasts.

As an improvement, and based on real call center data analysis, Aldor-Noiman et al. (2009) proposed the following linear mixed-effects (ME) model:

$$
Y_{i, j}=\alpha_{d_{i}}+\beta_{j}+\theta_{d_{i}, j}+\gamma_{i}+\epsilon_{i, j},
$$

where $\gamma_{i}$ denotes the daily volume deviation from the fixed weekday effect on day $i$. Then, $\gamma_{i}$ is the random effect on day $i$. Let $G$ denote the $D \times D$ covariance matrix for the sequence of random effects. The random effects, $\gamma_{i}$, are identically normally distributed with expected value $E[\gamma]=0$ and variance $\operatorname{Var}[\gamma]=\sigma_{G}^{2}$. The authors assume that these random effects follow an AR(1) process. Considering an AR(1) covariance structure for $G$ is both useful and computationally effective, because it requires the estimation of only two parameters, $\sigma_{G}$ and $\rho_{G}$. The residuals $\epsilon_{i, j}$ are also assumed to have an $\operatorname{AR}(1)$ structure within each day. As such, this model captures both interday and intraday dependencies in the data. Brown et al. (2005) proposed an earlier version of this model, also based on call-center data, without intraday correlations and without special-day effects.

In Ibrahim and L'Ecuyer (2013), the authors extended this ME model to two bivariate ME models for the joint distribution of the arrival counts to two separate queues, which exploit correlations between different call types. These models account for the dependence between the two call types by assuming that the vectors of random effects or the vectors of residuals across call types are correlated multinormal. This corresponds to using a normal copula; see Kim et al. (2012). The choice of copula can have a significant impact on performance measures in call centers, because of the strong effect of tail dependence on the quality of service Jaoua et al. (2013). A strong upper tail dependence for certain call types, for example, means that very large call volumes tend to arrive together for these call types. When this happens, this produces very large overloads.

To reduce the dimensionality of the vectors $\left(Y_{i, 1}, \ldots, Y_{i, P}\right)$, Shen and Huang (2005) proposed the use of singular-value decomposition to define a small number of vectors whose linear transformations capture most of the information relevant for prediction. Based on this, Shen and Huang (2008b) then developed a dynamic updating method for the distributional forecasts of arrival rates. Shen and Huang (2008a) proposed a method to forecast the latent rate profiles of a time series of inhomogeneous Poisson processes to enable forecasting future arrival rates based on a series of observed arrival counts.

Aktekin and Soyer (2011) recently proposed a model based on a Poisson-Gamma process, where $\Lambda_{i, j}=W_{i, j} \lambda_{i, j}$ for fixed $\lambda_{i, j}$ 's, and where the multiplicative factors $W_{i, j}$ have a gamma distribution and obey a gamma process. Soyer and Tarimcilar (2008) analyzed the effect of advertisement campaigns on call arrivals. Theirs is a Bayesian analysis where they model the Poisson 
rate function using a mixed model approach. This mixed model is shown to be superior to using a fixed-effects model instead. Weinberg et al. (2007) propose an adaptation of Brown et al. (2005) to enable it to update the forecasts of a day defined from the previous days using newly available observations during this day.

Weinberg et al. (2007) also used Bayesian techniques in their forecasts. They exploited the (normal) square-root transformed counts to include conjugate multivariate normal priors, with specific covariance structures. They used Gibbs sampling and the Metropolis Hastings algorithm to sample from the forecast distributions, which unfortunately requires long computational times. Moreover, it is unclear how to incorporate exogenous covariates in such a model.

In the empirical analysis of Taylor (2008), several time-series models are compared including Autoregressive Moving Average (ARMA) model and Holt-Winters exponential smoothing models with multiple seasonal patterns. The latter method was adapted by Taylor (2003) for modeling both the intraday and intraweek cycles in intraday data. In Taylor (2012), Taylor extended his model and considered the density forecasting of call arrival rates. To this aim, he developed a new Holt-Winters Poisson count data model with a gamma distributed stochastic arrival rate. He showed that this new model outperformed the basic Holt-Winters smoothing model. Shen (2010a) comments about Taylor's work, highlighting the difference between modeling arrivals as a single time series, and as a vector time series where each day is modeled as a component of that vector.

\subsection{Models Over a Single Day}

In this section, we focus on modeling arrivals over a single day. The day is divided into $p$ time periods. We denote by $\mathbf{X}=\left(X_{1}, \ldots, X_{p}\right)$ the vector of arrival counts in those periods.

It is commonly assumed that intraday arrivals follow a Poisson process with a random arrival rate. Whitt (1999a) proposed to do that by starting with a deterministic arrival rate function $\left\{\lambda(t), t_{0} \leq t \leq t_{\mathrm{e}}\right\}$, where $t_{0}$ and $t_{\mathrm{e}}$ are the opening and closing times of the call center for the considered day, and to multiply this function by a random variable $W$ with mean $\mathbb{E}[W]=1$, called the busyness factor for that day. The (random) arrival rate process for that day is then $\Lambda=\left\{\Lambda(t)=W \lambda(t), t_{0} \leq t \leq t_{\mathrm{e}}\right\}$.

Under this model, the arrival rates at any two given times are perfectly correlated, and $\operatorname{Corr}\left[\Lambda_{j}, \Lambda_{k}\right]=$ 1 for all $j, k$. We also expect the $X_{j}$ 's to be strongly correlated. More specifically, let $I_{j}$ denote the time interval of period $j$, let $\bar{\lambda}_{j}=\int_{I_{j}} \lambda(t) \mathrm{d} t$, and let $X_{j}$ be the number of arrivals in $I_{j}$. Using variance and expectation decompositions, one can find that $\operatorname{Var}\left[X_{j}\right]=\bar{\lambda}_{j}\left(1+\left(1+\bar{\lambda}_{j}\right) \operatorname{Var}[W]\right)$, i.e. (3.12) in Whitt (1999a), and for $j \neq k$ :

$$
\operatorname{Corr}\left[X_{j}, X_{k}\right]=\operatorname{Var}[W]\left[\left(\operatorname{Var}[W]+1 / \bar{\lambda}_{j}\right)\left(\operatorname{Var}[W]+1 / \bar{\lambda}_{k}\right)\right]^{-1 / 2} .
$$

This correlation is zero when $\operatorname{Var}[W]=0$ (a deterministic rate) and approaches 1 when $\operatorname{Var}[W] \rightarrow \infty$. Avramidis et al. (2004) studied this model in the special situation where $W$ has a gamma distribution with $\mathbb{E}[W]=1$ and $\operatorname{Var}[W]=1 / \gamma$. Then, each $\Lambda_{j}$ has a gamma distribution, and the $X_{j}$ 's have a negative multinomial distribution, whose parameters are easy to be estimated. Furthermore, the variance of the arrival counts can be made arbitrarily large by decreasing $\gamma$ toward zero. The model's flexibility is rather limited, because given the $\bar{\lambda}_{j}$ 's, $\operatorname{Var}\left[X_{j}\right]$ and $\operatorname{Corr}\left[X_{j}, X_{k}\right]$ for $j \neq k$ are all determined by a single parameter value, namely 
$\operatorname{Var}[W]$. In an attempt to increase the flexibility of the covariance matrix $\operatorname{Cov}[\mathbf{X}]$, and in particular to enable a reduction of the correlations, Avramidis et al. (2004) introduced two different models for $\mathbf{X}$, based on the multivariate Dirichlet distribution.

Jongbloed and Koole (2001) examined a similar model, but with independent busyness factors, one for each period of the day. Under their model, the $\Lambda_{j}$ 's are independent, as are the $X_{j}$ 's, which is inconsistent with intraday dependence of call center arrivals. Channouf (2008) considered a variant of the model where $\lambda(t)$ is defined by a cubic spline over the day, with a fixed set of knots, and also shows how to estimate model parameters. This can provide a smoother (perhaps more realistic) model of the arrival rate. Channouf (2008) and Channouf and L'Ecuyer (2012) proposed models that account for time dependence, overdispersion, and intraday dependencies with much more flexibility to match the correlations between the $X_{j}$ 's, by using a normal copula to specify the dependence structure between these counts. In principle, similar copula models could be developed for the vector of arrival rates, $\left(\Lambda_{1}, \ldots, \Lambda_{p}\right)$, instead of for the vector of counts. Oreshkin et al. (2014) examined the relationship between modeling for the vector of counts and for the vector of rates. In particular, they gave explicit formulas for the relationship between the correlation between rates and that between counts in two given periods, which implied that for a given correlation between rates, the correlation between counts is much smaller in low traffic than in high traffic.

\section{Case Studies}

In this section, we present empirical results from a case study using real data collected at a Canadian call center as described in Section 2. We use the data based on two call types and 200 consecutive workdays (excluding weekends). Four methods (Aldor-Noiman et al., 2009), Ibrahim and L'Ecuyer (2013) and Gans et al. (2015) to forecast arrival counts based on 6 week historical data are implemented in the study for each call type:

- MU: the multiplicative univariate forecasting model in Gans et al. (2015)

- ME: univariate mixed-effects model in Aldor-Noiman et al. (2009)

- BME1: bivariate mixed-effects model in Ibrahim and L'Ecuyer (2013)

- BME2: bivariate mixed-effects model in Ibrahim and L'Ecuyer (2013).

Each method is applied to the data and we assess the out-of-sample forecasting accuracy of each method. To compare different models, we use the Root Mean Squared Error (RMSE) to assess the point forecast accuracy as defined below:

$$
\mathrm{RMSE}=\sqrt{\frac{1}{K} \sum_{i, j}\left(X_{i, j}-\hat{X}_{i, j}\right)^{2}},
$$

where $\hat{X}_{i, j}$ is the predicted value of $X_{i, j}$ by the model, and $K$ is the total number of predictions. We also report the coverage probability for the $95 \%$ prediction interval to evaluate the forecasting distribution, which is defined as:

$$
\text { Cover }=\frac{1}{K} \sum_{i, j} \mathbb{I}\left(X_{i, j} \in\left(\hat{L}_{i, j}, \hat{U}_{i, j}\right)\right)
$$

where $\left(\hat{L}_{i, j}, \hat{U}_{i, j}\right)$ is the $95 \%$ prediction interval for $X_{i, j}$ given by the model. 
Tables 4 and 5 summarize the comparisons among the four methods. For both call types, ME is most accurate in point forecasts in most scenarios. BME1 and BME2 have better coverage probability when the leading period is one day or one week, and MU has better coverage probability when the leading period is 2 weeks.

Our numerical results serve to illustrate the complexity of the forecasting problem. Indeed, depending on the lead-time of interest, and on the specific criterion in mind, different models may be more appropriate, as shown here.

Selecting the "best" forecasting method ultimately depends on the specifics of the problem at hand, and an adequate measurement error. Proposing alternative arrival models that capture different features in the data are key to gaining a deeper understanding of the complexities of the call-arrival process.

\begin{tabular}{|l|l|r|r|r|r|}
\hline \multicolumn{7}{|c|}{ Type A } \\
\hline & & MU & ME & BME1 & BME2 \\
\hline 1 day ahead forecast & RMSE & 23.51 & 21.76 & 22.59 & 22.69 \\
& Cover & 0.89 & 0.91 & 0.93 & 0.93 \\
\hline 1 week ahead forecast & RMSE & 30.63 & 29.59 & 31.37 & 31.10 \\
& Cover & 0.88 & 0.88 & 0.88 & 0.86 \\
\hline 2 weeks ahead forecast & RMSE & 37.64 & 37.51 & 38.04 & 37.07 \\
& Cover & 0.84 & 0.82 & 0.80 & 0.79 \\
\hline
\end{tabular}

Table 4: Forecasting comparison among five methods for call type A.

\begin{tabular}{|l|l|r|r|r|r|}
\hline \multicolumn{7}{|c|}{ Type B } \\
\hline & & MU & ME & BME1 & BME2 \\
\hline 1 day ahead forecast & RMSE & 16.80 & 16.31 & 16.46 & 16.49 \\
& Cover & 0.93 & 0.92 & 0.95 & 0.95 \\
\hline 1 week ahead forecast & RMSE & 18.55 & 17.95 & 17.99 & 18.12 \\
& Cover & 0.95 & 0.91 & 0.94 & 0.94 \\
\hline 2 weeks ahead forecast & RMSE & 21.05 & 20.55 & 20.81 & 20.75 \\
& Cover & 0.93 & 0.86 & 0.90 & 0.90 \\
\hline
\end{tabular}

Table 5: Forecasting comparison among five methods for call type B.

\section{Conclusions and Discussions}

Forecasting call center arrivals plays a crucial role in call center management such as determining appropriate staffing level, scheduling plan and routing policy. The call center arrival process is complex and has to be modeled appropriately to achieve better forecasting accuracy, and as a result, more efficient operational decisions.

In this survey paper, we reviewed the existing literature on modeling and forecasting call center arrivals. We also conducted a case study to evaluate several recently proposed forecasting methods with real-life call center data.

An interesting future research direction is to extend the existing forecasting models or develop new models to forecast more than two call types simultaneously. As some stochastic optimization models for staffing and scheduling rely on the joint forecasting distribution of multiple 
types of arrivals, such multi-type forecasting models with full distributional forecasts have the potential to better meet the quality of service level and improve operational efficiency.

Another research question worth pursuing is to examine the operational impact of improved forecasts, as most existing literature about call center forecasting evaluate forecasting approaches based on only traditional statistical measures such as the RMSE and coverage probability without looking at how those improved forecasting models affect call center operations. By looking at the operational effect of forecasting models, managers can obtain more insight regarding forecasting model selection and system performance evaluation. Gans et al. (2015) have tried to tackle this problem for one call type. More research is needed in this direction.

In addition, different objectives of call center management may require minimizing different measures of forecasting errors. The question of what appropriate forecasting error measurement to choose and how it relates to call center decision making has not been well investigated. Ding and Koole (2015) show the optimal error measurement for minimizing the initial staffing costs plus the traffic management costs. However, the optimal error measurement for other managerial objectives remains unclear and needs to be studied.

Although there has been a lot of progress made in developing sophisticated forecasting methods, there remains a large gap between academic research and current industry practice, as discussed in Koole (2013). For example, most call center forecasting in practice is done using Excel, implementing simple decomposition based approaches; most workforce management (WFM) tools focus on scheduling, with limited functionality in forecasting. It remains critical for call center practice to incorporate advanced forecasting methods in such WFM tools.

We also want to point out that, besides call arrivals, there are other factors that need to be forecasted for efficient call center management, such as average handling time (AHT), workload, and even absenteeism. The relevant literature is rather sparse at this point. For example, AldorNoiman et al. (2009) used mixed effects models to forecast workload, while Gans et al. (2010) and Ibrahim et al. (2015) offered initial attempts at identifying factors that affect agent productivity, which can help with predicting the AHT.

\section{Acknowledgement}

We thank two anonymous referees and the editor for many helpful comments and suggestions. This work has been partially supported by grants from NSERC-Canada and Hydro-Quebec, and a Canada Research Chair, to P. L'Ecuyer, and from US-NSF (DMS-1106912, DMS-1407655), The Xerox Foundation, and HKU Stanley Ho Alumni Challenge Fund to H. Shen.

\section{References}

Akşin, O. Z., Armony, M., Mehrotra, V., 2007. The modern call center: A multi-disciplinary perspective on operations management research. Production and Operations Management $16(6), 665-688$.

Aktekin, T., Soyer, R., 2011. Call center arrival modeling: A Bayesian state space approach. Naval Research Logistics 58 (1), 28-42.

Aldor-Noiman, S., Feigin, P., Mandelbaum, A., 2009. Workload forecasting for a call center: Methodology and a case study. Annals of Applied Statistics 3, 1403-1447. 
Andrews, B., Cunningham, S. M., 1995. L.L. Bean improves call-center forecasting. Interfaces $25,1-13$.

Antipov, A., Maede, N., 2002. Forecasting call frequency at a financial services call centre. Journal of the Operational Research Society 53, 953-960.

Avramidis, A. N., Deslauriers, A., L'Ecuyer, P., 2004. Modeling daily arrivals to a telephone call center. Management Science 50 (7), 896-908.

Avramidis, A. N., L'Ecuyer, P., 2005. Modeling and simulation of call centers. In: Kuhl, M. E., Steiger, N. M., Armstrong, F. B., Joines, J. A. (Eds.), Proceedings of the 2005 Winter Simulation Conference. IEEE Press, pp. 144-152.

Barbour, A. D., Holst, L., Janson, S., 1992. Poisson Approximation. Oxford University Press, Oxford, U.K.

Bianchi, L., Jarrett, J., Hanumara, R. C., 1998. Improving forecasting for telemarketing centers by ARIMA modeling with intervention. International Journal of Forecasting 14, 497-504.

Brown, L., Cai, T., Zhang, R., Zhao, L., Zhou, H., 2010. The root-unroot algorithm for density estimation as implemented via wavelet block thresholding. Probability Theory and Related Fields 146, 401-433.

Brown, L., Gans, N., Mandelbaum, A., Sakov, A., Shen, H., Zeltyn, S., Zhao, L., 2005. Statistical analysis of a telephone call center: A queueing-science perspective. Journal of the American Statistical Association 100, 36-50.

Cez̧ik, M. T., L'Ecuyer, P., 2008. Staffing multiskill call centers via linear programming and simulation. Management Science 54 (2), 310-323.

Channouf, N., 2008. Modélisation et optimisation d'un centre d'appels téléphoniques: étude du processus d'arrivée. Ph.D. thesis, Département d'Informatique et de Recherche Opérationnelle, Université de Montréal, Canada.

Channouf, N., L'Ecuyer, P., 2012. A normal copula model for the arrival process in a call center. International Transactions in Operational Research 19, 771-787.

Channouf, N., L'Ecuyer, P., Ingolfsson, A., Avramidis, A. N., 2007. The application of forecasting techniques to modeling emergency medical system calls in Calgary, Alberta. Health Care Management Science 10 (1), 25-45.

Ding, S., Koole, G., 2015. Optimal call center forecasting and staffing under arrival rate uncertainty. Working paper.

Ding, S., Koole, G., Mei, R. V. D., 2015. On the estimation of the true demand in call centers with redials and reconnects. European Joural of Operations Research 246 (1), 250-262.

Gans, N., Koole, G., Mandelbaum, A., 2003. Telephone call centers: Tutorial, review, and research prospects. Manufacturing \& Service Operations Management 5, 79-141.

Gans, N., Liu, N., Mandelbaum, A., Shen, H., Ye, H., 2010. Service times in call centers: Agent heterogeneity and learning with some operational consequences. In: Borrowing Strength: Theory Powering Applications - A Festschrift for Lawrence D. Brown. Vol. 6. IMS Collections, Beachwood, OH, pp. 99-123. 
Gans, N., Shen, H., Zhou, Y. P., Korolev, N., McCord, A., Ristock, H., 2015. Parametric forecasting and stochastic programming models for call-center workforce scheduling. Manufacturing \& Service Operations Management, in press.

Garnett, O., Mandelbaum, A., Reiman, M., 2002. Designing a call center with impatient customers. Manufacturing \& Service Operations Management 4 (3), 208-227.

Glynn, P., Hong, L. J., Zhang, X., 2014. Modeling call center arrivals: a tale of three timescales. Work in progress.

Green, L. V., Kolesar, P. J., Whitt, W., 2007. Coping with time-varying demand when setting staffing requirements for service system. Production and Operations Management 16 (1), $13-39$.

Hyndman, R. J., Koehler, A. B., Ord, J. K., Snyder, R. D., 2008. Forecasting with Exponential Smoothing: The State Space Approach. Springer.

Ibrahim, R., L'Ecuyer, P., 2013. Forecasting call center arrivals: Fixed-effects, mixed-effects, and bivariate models. Manufacturing \& Services Operations Management 15 (1), 72-85.

Ibrahim, R., L'Ecuyer, P., Régnard, N., Shen, H., 2012. On the modeling and forecasting of call center arrivals. In: Proceedings of the 2012 Winter Simulation Conference. IEEE Press, pp. $1-12$.

Ibrahim, R., L'Ecuyer, P., Shen, H., Thiongane, M., 2015. Inter-dependent, heterogeneous, and time-varying service-time distributions in call centers. Under review.

Jaoua, A., L'Ecuyer, P., Delorme, L., 2013. Call type dependence in multiskill call centers. Simulation 89 (6), 722-734.

Jongbloed, G., Koole, G., 2001. Managing uncertainty in call centers using Poisson mixtures. Applied Stochastic Models in Business and Industry 17, 307-318.

Kim, S. H., Whitt, W., 2014a. Are call center and hospital arrivals well modeled by nonhomogeneous poisson processes? Manufacturing \& Service Operations Management 16 (3), 464-480.

Kim, S. H., Whitt, W., 2014b. Choosing arrival process models for service systems: Tests of a nonhomogeneous poisson process. Naval Research Logistics 61 (1), 66-90.

Kim, T., Kenkel, P., Brorsen, B. W., 2012. Forecasting hourly peak call volume for a rural electric cooperative call center. Journal of Forecasting 31, 314-329.

Koole, G., 2013. Call Center Optimization. MG Books.

Landon, J., Ruggeri, F., Soyer, R., Tarimcilar, M. M., 2010. Modeling latent sources in call center arrival data. European Journal of Operations Research 204 (3), 597-603.

Liao, S., Delft, C. V., Koole, G., Jouini, O., 2012. Staffing a call center with uncertain nonstationary arrival rate and flexibility. OR Spectrum 34 (3), 691-721.

Mabert, V. A., 1985. Short interval forecasting of emergency phone call (911) work loads. Journal of Operations Management 5 (3), 259-271.

Maman, S., Mandelbaum, A., Whitt, W., Zeltyn, S., 2015. Queues with random arrival rates: Inference, modelling and asymptotics (c-staffing). Work in progress. 
Oreshkin, B. N., Regnard, N., L'Ecuyer, P., 2014. Rate-based daily arrival process models with application to call centers. Working paper.

Shen, H., 2010a. Exponentially weighted methods for forecasting intraday time series with multiple seasonal cycles: Comments. International Journal of Forecasting 58, 652-654.

Shen, H., 2010b. Statistical analysis of call-center operational data: Forecasting call arrivals, and analyzing customer patience and agent service. In: Cochran, J. J. (Ed.), Wiley Encyclopedia of Operations Research and Management Science. John Wiley.

Shen, H., Huang, J. Z., 2005. Analysis of call centre arrival data using singular value decomposition. Applied Stochastic Models in Business and Industry 21, 251-263.

Shen, H., Huang, J. Z., 2008a. Forecasting time series of inhomogeneous Poisson processes with application to call center workforce management. Annals of Applied Statistics 2 (2), 601-623.

Shen, H., Huang, J. Z., 2008b. Interday forecasting and intraday updating of call center arrivals. Manufacturing \& Service Operations Management 10 (3), 391-410.

Soyer, R., Tarimcilar, M. M., 2008. Modeling and analysis of call center arrival data: A Bayesian approach. Management Science 54 (2), 266-278.

Steckley, S. G., Henderson, S. G., Mehrotra, V., 2004. Service system planning in the presence of a random arrival rate. Submitted.

Steckley, S. G., Henderson, S. G., Mehrotra, V., 2005. Performance measures for service systems with a random arrival rate. In: Kuhl, M. E., Steiger, N. M., Armstrong, F. B., Joines, J. A. (Eds.), Proceedings of the 2005 Winter Simulation Conference. IEEE Press, pp. 566-575.

Steckley, S. G., Henderson, S. G., Mehrotra, V., 2009. Forecast errors in service systems. Probability in the Engineering and Informational Sciences 23 (2), 305-332.

Tanir, O., Booth, R. J., 1999. Call center simulation in Bell Canada. In: Proceedings of the 1999 Winter Simulation Conference. IEEE Press, Piscataway, New Jersey, pp. 1640-1647.

Taylor, J. W., 2003. Short-term electricity demand forecasting using double seasonal exponential smoothing. Journal of Operational Research Society 54, 799-805.

Taylor, J. W., 2008. A comparison of univariate time series methods for forecasting intraday arrivals at a call center. Management Science 54 (2), 253-265.

Taylor, J. W., 2012. Density forecasting of intraday call center arrivals using models based on exponential smoothing. Management Science 58 (3), 534-549.

Thompson, H. E., Tiao, G. C., 1971. Analysis of telephone data: A case study of forecasting seasonal time series. The Bell Journal of Economics and Management Science 2 (2).

Wallace, R. B., Whitt, W., 2005. A staffing algorithm for call centers with skill-based routing. Manufacturing \& Service Operations Management 7 (4), 276-294.

Weinberg, J., Brown, L. D., Stroud, J. R., 2007. Bayesian forecasting of an inhomogeneous Poisson process with applications to call center data. Journal of the American Statistical Association 102 (480), 1185-1198.

Whitt, W., 1999a. Dynamic staffing in a telephone call center aiming to immediately answer all calls. Operations Research Letters 24, 205-212. 
Whitt, W., 1999b. Improving service by informing customers about anticipated delays. Management Science 45 (2), 192-207.

Zhang, X., Hong, L. J., Zhang, J., 2014. Scaling and modeling of call center arrivals. In: Proceedings of the 2014 Winter Simulation Conference. IEEE, pp. 476-485. 